\title{
Enantioselective Synthesis of Bioactive Molecules Related to Plant Protection and Physiology
}

\author{
Takeshi Kitahara and Hidenori Watanabe
}

Department of Applied Biological Chemistry, Graduate School of Agricultural and Biological Sciences

The University of Tokyo,

\begin{abstract}
Enantioselective synthesis of bioregulators with remarkable biological activities has been accomplished. The target molecules are plant growth regulators (methyl epijasmonate and analogs, pironetin), plant pathogenic toxins (oxygenated eremophilanes, sorokinianin) and substances related to plant protection (tanabalin; insect antifeedant, mugineic acid and analogs; phytosiderophore).
\end{abstract}

\section{Introduction}

Basic research on plant protection and plant physiology is becoming more and more important in order to increase plant-production which is essential for human life and amenity worldwide in the coming 21 st century. In this context, we have been studying the synthesis of substances with remarkable bioactivities, such as plant growth regulation, plant nutrition and plant protection, to produce substantial quantities of samples for biological and physiological studies, to evaluate their structure-activity relationships and to create novel useful substances with high activities. We describe herein the enantioselective synthesis of these substances shown in Fig. 1.

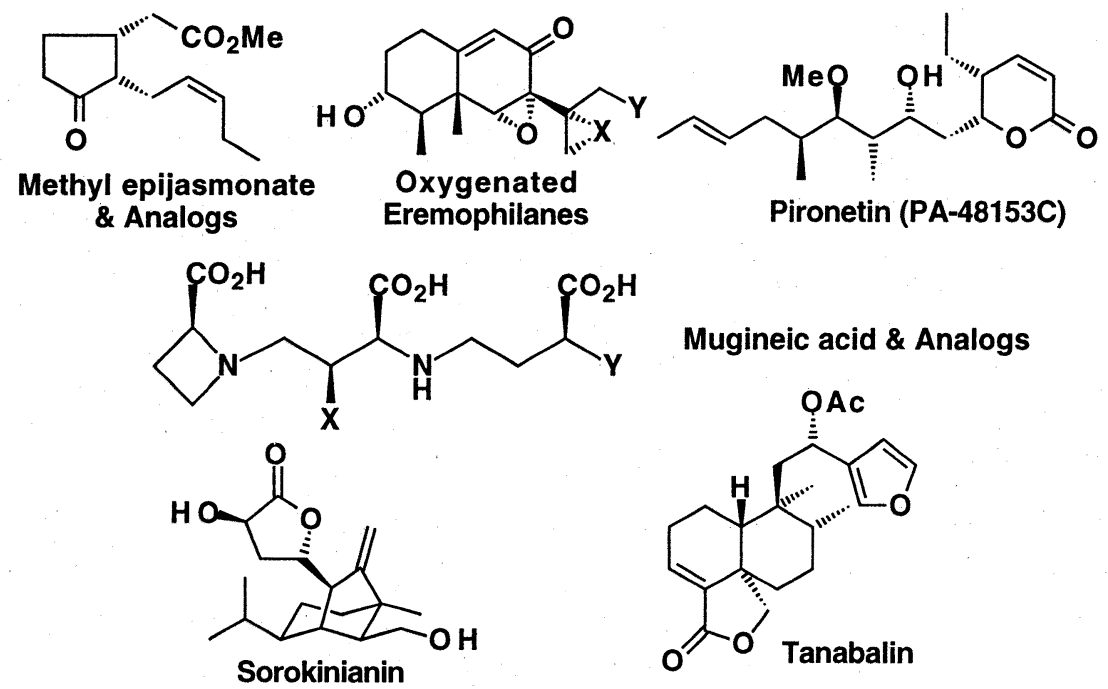

Fig. 1 Structures of Our Synthetic Targets 


\section{Synthesis of Methyl Epijasmonate and Related Analogs}

Jasmine oil manufactured from jasmine flowers is a well-known fragrant material for practical use. In 1962, Demole et al. identified methyl jasmonate (MJ) 1 as a minor but a key fragrant component of jasmine oil (ref. 1). Later, the corresponding acid analog of 1, jasmonic acid, was isolated from the culture broth of plant pathogenic fungus, Lasiodoplodia theobromae, as a plant growth inhibitor (ref. 2). It was found that methyl jasmonate itself also had similar activity and jasmonate analogs have been postulated as potent plant growth regulators. Thus, the importance of these compounds has been well recognized not only in perfumery industry but also in the field of plant physiology.

In relation to their remarkable biological functions, investigations to detect and isolate these jasmonoids from natural sources have actively been pursued. Those studies have culminated in the characterization of the less stable isomer of $\mathbf{1}$, methyl epijasmonate $\mathbf{2}$, from the pheromone of the male Oriental fruit moth, Grapholitra molesta (ref. 3) and so on. More recently, similar cis-analogs, $\beta$-Dglucopyranosyltuberonic acid 3 (ref. 4) and its methyl ester 4 (ref. 5) were detected in potato and other related species as tuber-inducing factors. In addition to these glycosides, the aglycone, methyl tuberonate $\mathbf{5}$, methyl epijasmonate 2 and other analogs exhibited similar biological activities. Quantitative evaluation of these substances, however, has not been undertaken extensively, because of the shortage of the pure materials.

From these standpoints of view, we synthesized chemically and optically pure jasmonoids not only for practical use but also to provide a biological tool. Here we focus our discussion on the synthesis of a more labile cis-series.

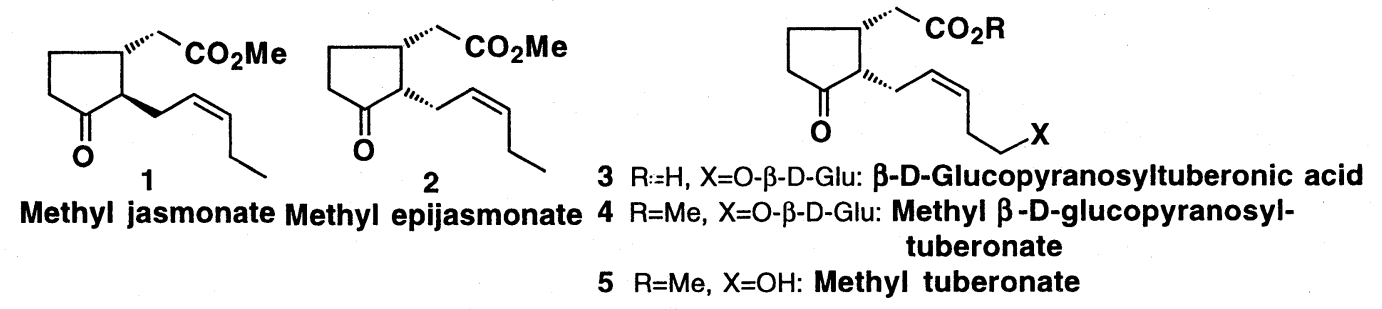

Fig. 2 Structures of Jasmonoids

\subsection{Synthesis of Both Enantiomers of Methyl Epijasmonate}

Enantioselective synthesis of methyl epijasmonate (MEJ) was first reported by Helmchen and coworkers using asymmetric Diels-Alder reaction (ref. 6). Almost at the same time, we succeeded in the efficient synthesis of both enantiomers of MEJ starting from both enantiomers of the known bicyclic lactone 6 which is readily available as a starting material for prostaglandin synthesis. DIBAL reduction of the (-)-lactone, (-)-6, was followed by acetal formation and successive epoxidation with MCPBA gave the epoxide 7. Regiospecific reduction of the oxirane ring and then PDC oxidation gave the ketone 8, which was converted to the desired ester 9 with an acetate side chain. Stereospecific hydrogenation from the $\beta$ face was expected because of the nature of the 2-oxabicyclo[3.3.0]octane system, but this was proved at a later stage. Acid treatment of 9 and the following salt-free Wittig reaction gave the bridged lactone $\mathbf{1 0}$ with the desired stereochemistry via concomitant cyclization. The formation of the lactone ring was unambiguous proof of stereospecific hydrogenation from the $\beta$-face. The following three-step conversion gave natural (+)-2 in $26 \%$ overall yield through 12 steps. In order to prevent isomerization to the transisomer during the final oxidation step, we found that two-phase oxidation with chromic acid in etherwater was suitable, giving the pure $c$ is-isomer. Starting from the other enantiomer, (+)-6, the unnatural antipode (-)-2 was obtained (ref. 7). 
<smiles>O=C1C[C@H]2C=CC[C@H]2O1</smiles>

i) DIBAL ii) $\mathrm{H}^{+} / \mathrm{MeOH}$

iii) $\mathrm{MCPBA}$

(75\%)

$(-)-6$<smiles>CO[C@H]1C[C@@H]2[C@@H](CC[C@@H]1CC(N)=O)C[C@H]2OC</smiles>

9

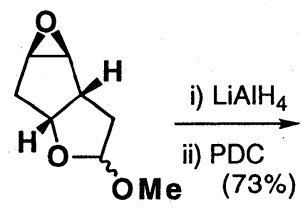

7

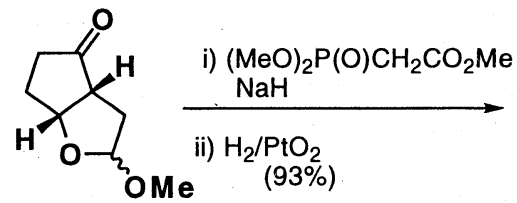

8

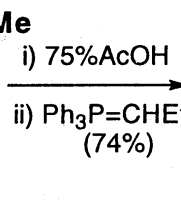
(74\%)<smiles>CC/C=C/CC1CC2CCC1OC(=O)C2</smiles>

10 i) $\mathrm{KOH} / \mathrm{MeOHaq}$. ii) $\mathrm{CH}_{2} \mathrm{~N}_{2}$

iii) $\mathrm{H}_{2} \mathrm{Cr}_{2} \mathrm{O}_{7} / \mathrm{Et}_{2} \mathrm{O}-\mathrm{H}_{2} \mathrm{O}$ $7 \mathrm{~min}(69 \%)$<smiles>CC/C=C\C[C@H]1C(=O)CC[C@@H]1CC(C)=O</smiles>

$(+)-2$

Fig. 3 Synthesis of (+)-Methyl Epijasmonate (Overall 12 steps, 26\%)

\subsection{Synthesis of Both Enantiomers of Methyl cis-(Z)-Dehydrojasmonate}

Using this strategy, related jasmonate analogs were synthesized with some modification. It was necessary to execute the Wittig reaction of the intermediary hemiacetal at room temperature, because the stable hemiacetal was not so reactive, resulting in the formation of a mixture of cis- and trans-olefin in the side chain (ca. 9:1). In order to improve this process, we employed a lactone-aldehyde. Thus, in the synthesis of both enantiomers of methyl cis-(Z)-dehydrojasmonate 11 (ref. 8), the key fragrant substance of an Asian orchid, Cymbidium goeringii (ref. 9), lactone thioacetal (-)-12 was prepared from the acetal 9. The Wittig reaction of the corresponding aldehyde at $-78^{\circ} \mathrm{C}$ gave phenylselenoolefin 13 and the cisselectivity was shown to be more than $98 \%$. Oxidative elimination of selenide gave the diene lactone 14 and the following conversion furnished the target molecule (+)-11. Dess-Martin oxidation was the reagent of choice for the final step giving 11 in excellent yield with no epimerization.<smiles>CO[C@H]1C[C@@H]2[C@@H](CCC[C@H]2C(=O)O)O1</smiles>

9

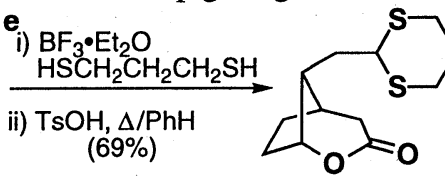

$(-)-12$ i) $\mathrm{HgO}-\mathrm{HgCl}_{2}$
ii) $\mathrm{Ph}_{3} \mathrm{P}=\mathrm{CHCH}_{2} \mathrm{CH}_{2} \mathrm{SePh}$
THF-HMPA, $-78^{\circ} \mathrm{C}$ (57\%)

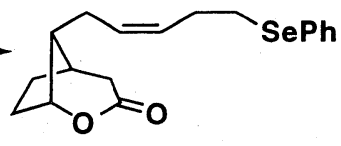

13<smiles>C=C/C=C\C[C@H]1C(=O)CC[C@H]1CCOC(C)=O</smiles>

Fig. 4 Synthesis of Methyl cis-(Z)-Dehydrojasmonate (Overall 15 steps, 17\%)

\subsection{Synthesis of Methyl Tuberonate}

We adopted a similar strategy for the synthesis of methyl tuberonate 5 using $\omega$ tetrahydropyranoxyphosphorane. In this case, however, after oxidation of cyclopentanol, the protecting group of the $\omega$-hydroxyl group had to be removed without epimerization of the cis-side chain. Unfortunately, hydrolysis of THP (70\% aqueous acetic acid, $\left.50^{\circ} \mathrm{C}, 60 \mathrm{~min}\right)$ or even 1-ethoxyethyl $(70 \%$ acetic acid aq., $50^{\circ} \mathrm{C}, 5 \mathrm{~min}$ ) caused epimerization to some extent. After several trials, we could optimize the route to the product without any epimerization. The Wittig product 16 was converted to the $\omega$ - 
trifluoroacetoxycyclopentanol 18 in 5 steps via exchange of the protecting groups. After Dess-Martin oxidation, simple treatment of the ketone 19 with methanol at room temperature without using any base or acid, gave pure methyl tuberonate $(+)-5$ via transesterification (refs. 10,11 ).<smiles>O=C1CC2C(CC3SCCCS3)CCC2O1</smiles>

$(-)-12$

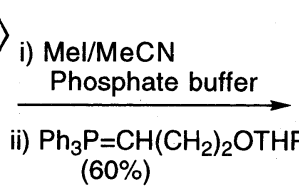

$(60 \%)$

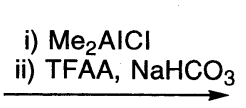

iii) HF-MeCNaq.

(44\%)

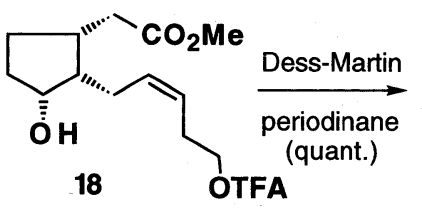

18

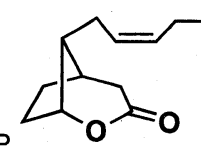

16

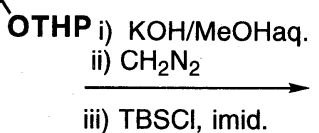

$(83 \%)$

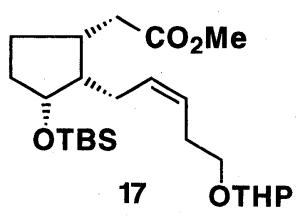

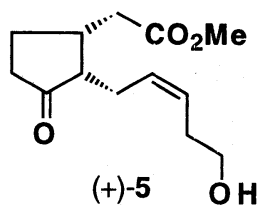

Fig. 5 Synthesis of Methyl Tuberonate (Overall 19 steps, $7 \%$ )

\subsection{Synthesis of Methyl $\beta$-D-Glucopyranosyltuberonate}

The intermediate $\mathbf{2 0}$ for the synthesis of 5 was glycosylated to give the protected glycoside $\mathbf{2 1}$. In order to synthesize methyl $\beta$-D-glucopyranosyltuberonate 4 , it was again necessary to remove the protecting groups of four hydroxyl groups on the glucosyl moiety without epimerization of the $\alpha$ substituent. We tried to use the protecting method developed for the synthesis of methyl tuberonate (vide supra), but in this case, trifluoroaceate was too labile to be retained safely during deprotection and oxidation steps. Selection of dichloroacetate as a protecting group overcame this difficulty and thus, acetyl groups of the glycoside $\mathbf{2 1}$ were replaced by dichloroacetyl groups to give the dichloroacetate $\mathbf{2 3}$. Again, simple methanolysis furnished the target glycoside 4 without any epimerization (ref. 11).

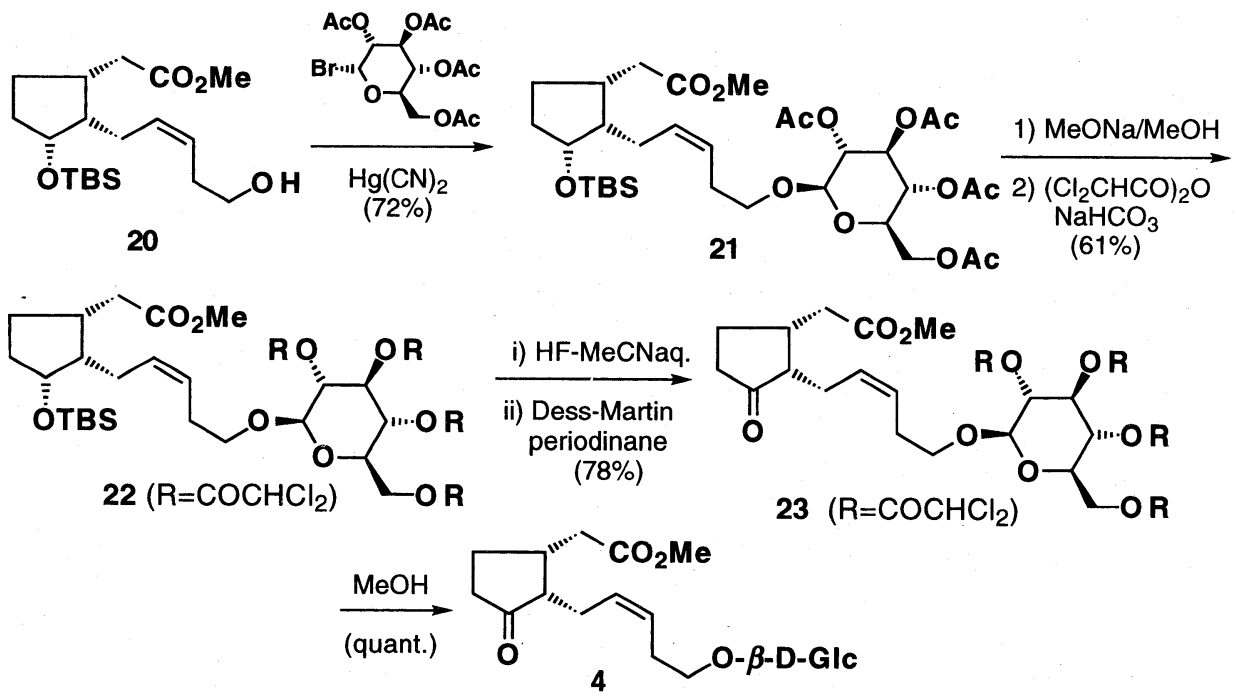

Fig. 6 Synthesis of Methyl $\beta$-D-Glucopyranosyltuberonate (Overall 21 steps, 6\%) 


\section{Synthesis of Oxygenated Eremophilanes: Phytotoxins}

In recent years, highly oxygenated eremophilane sesquiterpenes have been isolated from the microbial world. They possess remarkable bioactivities, such as phytotoxicity (compounds $\mathbf{2 4 ~ 2 8}$ ), plant hormone inhibitor (30) and so on (ref. 12). Among them, Sporogen-AO 124 is a microbial hormone with spore-inducing activity to fungus, Aspergillus oryzae (ref. 12a), and Bipolaroxin $\mathbf{2 8}$ is a hostselective phytotoxin against several weeds (ref. 12e). KM-01 is an inhibitor to brassinosteroids, which are now recognized as the sixth plant hormone (ref. 12g). Being aware of such interesting bioactivities, we have investigated the synthesis of these bioregulators.<smiles>[X]CC(=C)C12O[C@@H]1[C@]1(C)C(=CC2=O)CC[C@H](O)[C@@H]1C</smiles>

$24 \mathrm{X}=\mathrm{H}$ : Sporogen-AO 1 (13-Desoxyphomenone)

$25 \mathrm{X}=\mathrm{OH}$ : Phomenone<smiles>CCC(C)/C=C/C=C/C(=O)O[C@H]1C=CC2=CC(=O)[C@@H](O)C[C@]2(C)C1C</smiles>

29 Dendryphiellin C

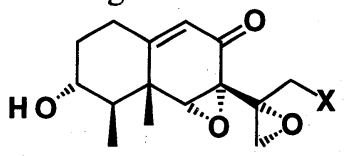

$26 \mathrm{X}=\mathrm{H}$ : Gigantenone $27 \mathrm{X}=\mathrm{OH}$ : Phaseolinone<smiles>C=C(C=O)[C@]1(O)C[C@]2(C)C(=CC1=O)C=C[C@H](O)[C@@H]2C</smiles>

28 Bipolaroxin<smiles>C=C(C=O)[C@]1(O)C[C@]2(C)C(=CC1=O)C=C[C@H](OC(=O)/C=C/C=C/[C@@H](C)CC)[C@@H]2C</smiles>

30 KM-01

Fig. 7 Highly Oxygenated Eremophilanes as Toxins and Inhibitors

\subsection{Preparation of a Chiral Building Block by Reduction with Baker's Yeast}

In 1985 , we succeeded in the efficient preparation of chiral building block 32 with suitable functional groups in the molecule by reduction of the known $\beta$-ketoester 31 (ref. 13) with baker's yeast. Optical purity of the resulting hydroxyester 32 was determined as its MTPA ester to be an extremely high 98.6\%e.e. This building block is now available in large quantity by simple operation (ref. 14).<smiles>O=C(OF)C1CCC2(CC1)OCCO2</smiles>

31

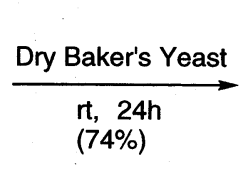

$(74 \%)$

Lo

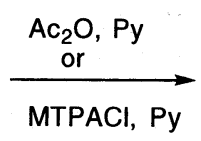

32<smiles>CCOC(=O)[C@H]1CC2(CC[C@H]1O)OCCO2</smiles>

33

$\mathrm{R}=\mathrm{Ac} ; \quad \mathrm{H}_{1}, \quad \mathrm{hhw}=7.0 \mathrm{~Hz}$

$\mathrm{R}=(S)-$, (R)-MTPA ; (98.6\%e.e.)

Fig. 8 Preparation of a Chiral Building Block by Reduction with Baker's Yeast

\subsection{Synthesis of Sporogen-AO 1: Sporogenic Hormone}

We reasoned that this building block 32 should be convertible to the properly substituted cyclohexanone 35, which would give the desired octalone 36 via Robinson annulation. In fact, this was realized via bicycloheptanone 34 prepared from 32 through 5 steps in excellent yield. The presence of the cyclopropane ring in 34 was essential to promote the Mitsunobu reaction smoothly to give the epimer with correct stereochemistry, which on reductive alkylation gave 35 regioselectively. Robinson annulation via regioselective formation of silyl enol ether gave octalone 36 . Thus, without using any blocking group, the octalone system was selectively constructed. Introduction of an acetyl group and a double bond afforded dione 37. Stereo- and regiospecific epoxidation at the doubly activated olefin and 
finally, Peterson-type methylenation gave sporogen-AO 124 (ref. 15).<smiles>CCOC(=O)[C@H]1CC2(CC[C@@H]1O)OCCO2</smiles>

32

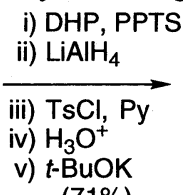

$(71 \%)$<smiles>O=C1CCC(O)C2CC12</smiles>

34

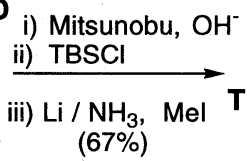

i) Mitsunobu, $\mathrm{OH}^{-}$ ii) $\mathrm{TBSCl}$ $(67 \%)$

iii) $\mathrm{Li} / \mathrm{NH}_{3}, \mathrm{Mel}$ TBSO"<smiles>C[C@H]1C(=O)CC[C@@H](O[AsH3])[C@@H]1C</smiles>

35 i) $\mathrm{TMSI} / \mathrm{HMDS}$

ii) $\mathrm{MVK}, \mathrm{BF}_{3}-\mathrm{Et}_{2} \mathrm{O}$

iii) pyrrolidine, $\Delta$ (54\%)

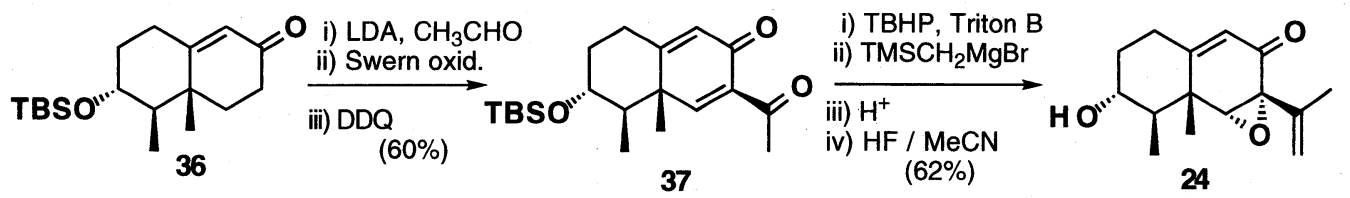

Fig. 9 Synthesis of (+)-Sporogen-AO 1 (Overall 18 steps, 10\%)

Using this strategy, we were able to synthesize phomenone $\mathbf{2 5}$, gigantenone 26 and phaseolinone 27 (ref. 16). Both enantiomers of phaseic acid, a metabolite of abscisic acid, were synthesized using $\mathbf{3 2}$ as a single chiral source (ref. 17). Just recently, we also succeeded in the synthesis of dendryphiellin $\mathrm{C}$ 29 (ref. 18). The synthesis of bipolaroxin 28 and $\mathrm{KM}-0130$ is due to be completed in the near future.

\section{Synthesis of Pironetin: Growth Inhibitor}

Pironetin (PA-48153C) 38 was isolated from the culture broth of Streptomyces sp. NK10958 (ref. 19) or Streptomyces prunicolor (ref. 20). It shows interesting bioactivity as a plant growth inhibitor, which might be applicable to the production of dwarf plants; it also contains potent immunosuppressive activity on the responses of both $\mathrm{T}$ and $\mathrm{B}$ lymphocytes to mitogens. While several syntheses of pironetin 38 have been already reported (ref. 21), we have recently completed its synthesis via a convergent route using our chiral building block 34 (ref. 22). We dissected the molecule at C6-C7 and employed the coupling of epoxide 40 with the dithiane 39 , which would be derivable from 34 .
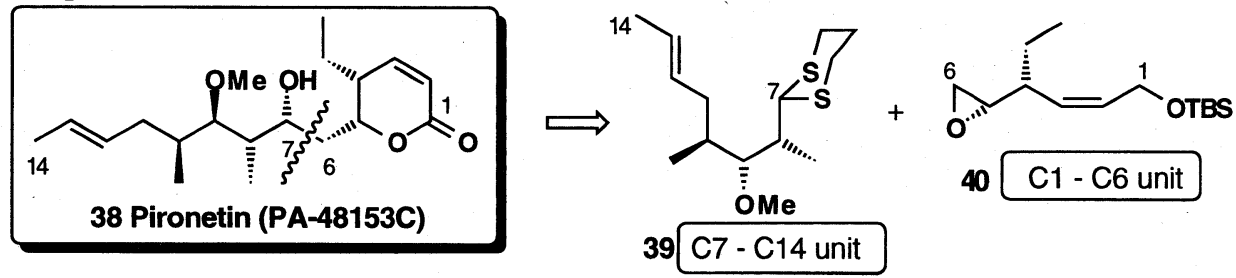

Fig. 10 Synthesis of Pironetin : Plan

The C1-C6 unit 40 was derived from the known epoxide 41 via opening with acetylenic alanate (ref. 23) and successive epoxide formation. Meanwhile, stereospecific methylation of the ketol $\mathbf{3 4}$ and selective reduction gave the desired isomer 43a after etherification. Stereochemistry was determined by $\mathrm{X}$-ray analysis of the crystalline diol. Oxidation followed by a two-step hydroxylation and then Birch reduction gave the expected ketol 44. Oxidative cleavage with lead tetraacetate and functional group manipulation afforded the aldehyde 45, which on Takai reaction (ref. 24) and thioacetalization gave the C7-C14 unit, the dithiane 39. Coupling reaction of 39 and 40 proceeded smoothly at $0^{\circ} \mathrm{C}$ in a short time to give the whole skeleton 46 . Prolonged reaction period resulted in extensive decomposition of the product. Cleavage of thioacetal and the following stereoselective reduction by Mori's procedure (ref. 25) gave anti-diol 47 a predominantly. Finally, desilylation and oxidation with manganese dioxide afforded pironetin 38 (ref. 22). 

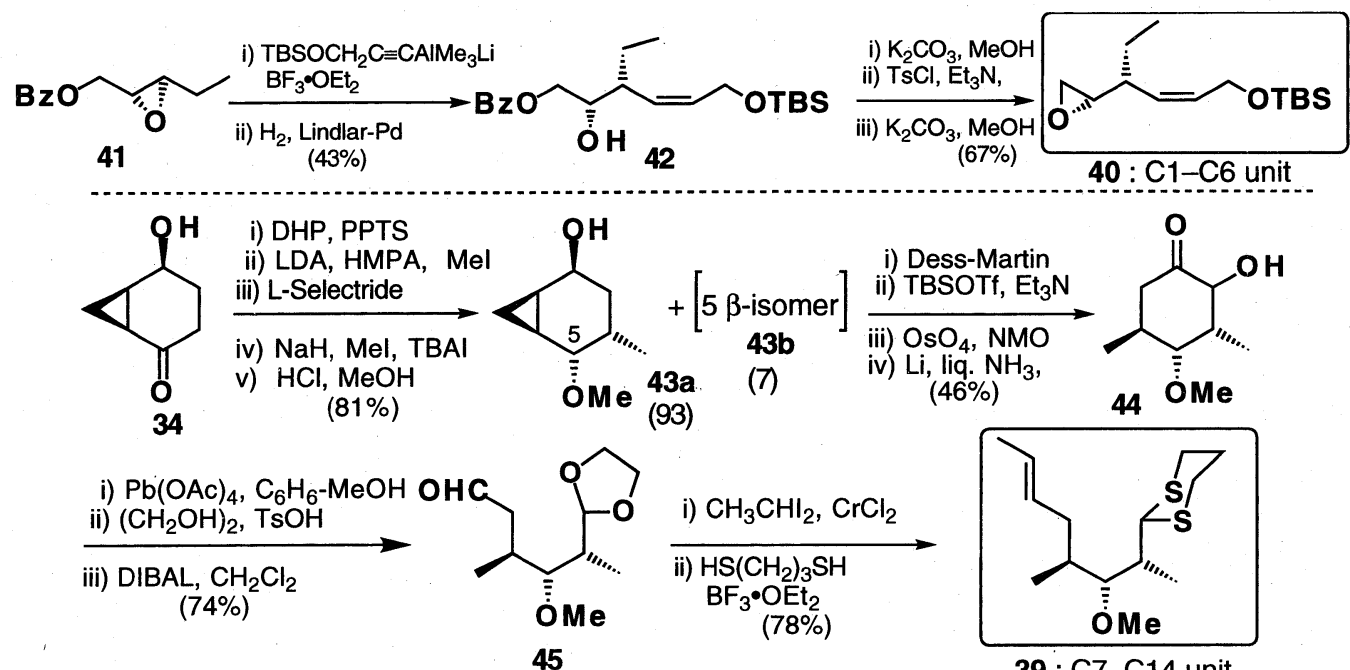

39 : C7-C14 unit

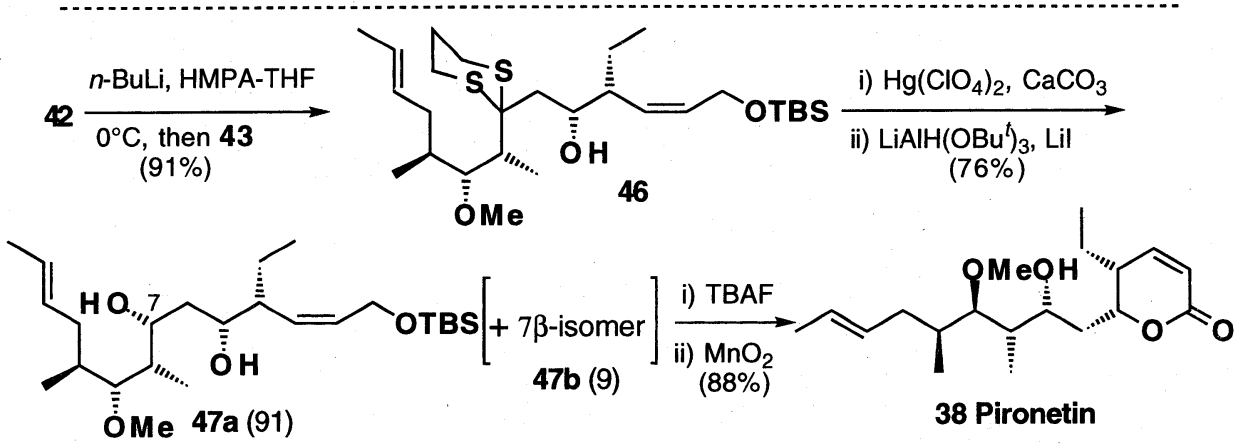

Fig. 11 Synthesis of Pironetin (Overall 19 steps, 12.9\%)

\section{Synthesis of 2'-Deoxymugineic Acid and Related Analogs: Phytosiderophores}

Mugineic acid and analogs 48 50 are well-known phytosiderophores, which are excreted from the root of grasses (rice, barley, wheat, etc.) under iron-deficient conditions to dissolve and absorb the ferric ion by chelation (ref. 26). Because of such remarkable activity, a number of syntheses have been reported to date (ref. 27). Nevertheless, physiologists still require large quantities of such phytosiderophores for field studies. We wanted to devise a versatile method both for large-scale production and for obtaining various analogs. In every synthesis ever known, reductive amination was employed for connecting the three units. Unstable aldehydes, however, had to be used for those processes and handling of basic intermediates was rather difficult. Our basic plan was to construct the skeleton as stable tripeptides $\mathbf{5 1}$<smiles>[Y]C(CCNC(C(=O)O)C([Y])CN1CCCC1)C(=O)O</smiles>

48 Mugineic acid : $\mathrm{X}=\mathrm{Y}=\mathrm{OH}$

49 2'-Deoxymugineic acid : $\mathrm{X}=\mathrm{H}, \mathrm{Y}=\mathrm{OH}$

50 Nicotianamine : $X=\mathrm{H}, Y=\mathrm{NH}_{2}$

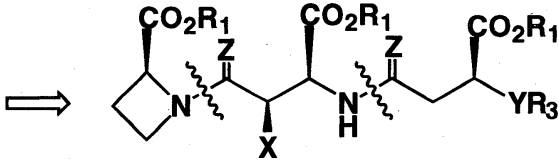

$51 \mathrm{Z}=\mathrm{O} ; 52 \mathrm{Z}=\mathrm{S}$

Fig. 12 Synthesis of Phytosiderophores : Plan 
and then to reduce the amide group chemoselectively via thioamide $\mathbf{5 2}$ without attacking other functionalities to give the targets $48 \sim 50$.

Azetidine carboxylate $\mathbf{5 3}$ was sequentially condensed with the corresponding amino acid derivatives 54 and 56 , which were derived from natural aspartic acid and malic acid, respectively, to give the desired tripeptide 57. Although direct borane reduction was not so chemoselective, treatment with Lawesson's reagent afforded thioamide $\mathbf{5 8}$ in good yield and successive desulfurization with Raney-nickel followed by deprotection furnished 2'-deoxymugineic acid 49. This expeditious process efficiently gave the target compound in excellent overall yield $27 \%$ through 7 steps (ref. 28). In the same manner, nicotianamine 50 was synthesized. The synthesis of mugineic acid $\mathbf{4 8}$ and avenic acid is also forthcoming (ref. 29). Since the azetidine ring and 2'-hydroxyl group of mugineic acid are not essential for the expression of activity, we are attempting to develop large-scale production of simpler analogs according to this scheme.
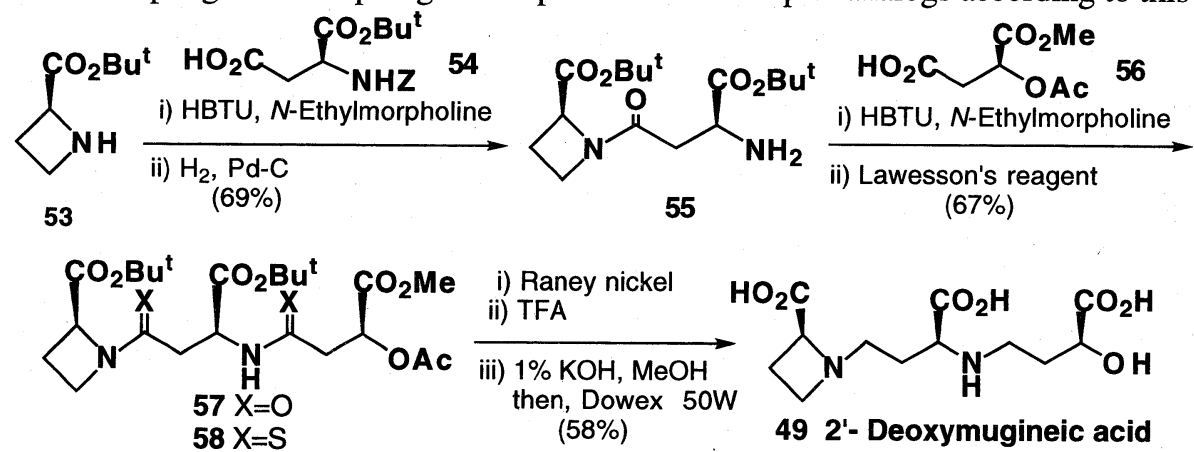<smiles>O=C(O)[C@H](CCN1C[C@H](C(=O)O)[C@@H]1C(=O)O)NCC[C@@H](O)C(=O)O</smiles>

Fig. 13 Synthesis of 2'-Deoxymugineic acid (Overall 7 steps, 27\%)

\section{Synthesis of Sorokinianin: Phytotoxin}

Sorokinianin 59 was isolated by Nakajima et al. from pathogenic fungus, Helminthosporium sorokiana, which infects Australian barley. It shows potent growth inhibition, and the relative stereochemistry was elucidated. Availability of the compound, however, was limited and the absolute configuration was unknown (ref. 30). We noticed its biological activity and decided to synthesize the enantiomer to elucidate the absolute configuration as well as to obtain substantial amount of the material. Its structure is similar to that of helminthosporal, which has already been synthesized (ref. 31), except that it contains an additional three-carbon unit as the hydroxylactone moiety. So, we selected a different and more accessible approach to reach 59 , namely, bicyclic ketone 60 , which should be derived from carvone 61, was employed as a key intermediate. As shown in Fig. 14, addition of $C_{1}$ - and the $C_{3}$-unit to C6and C7-position and introduction of oxygen to C8-methylene would afford sorokinianin.

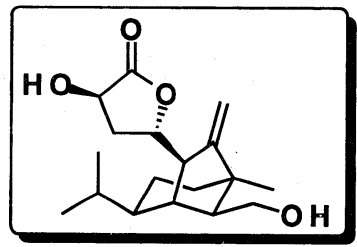

59 Sorokinianin

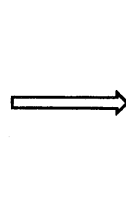

Fig. 14 Synthesis of Sorokinianin : Plan
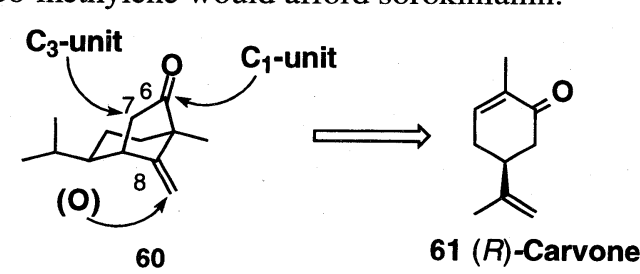

$(R)-(+)$-Carvone 61 was converted to ketoaldehyde 62 in three steps, which was treated with a base to equilibrate the mixture giving the desired bicyclic ketol with the equatorial isopropyl group predominantly (4:1). The mixture was separated after silylation to give pure 63 . The minor isomer with 
axial substituent was equilibrated by the same base treatment to give more 63 . The Wittig reaction and oxidation of the regenerated hydroxyl group gave the key intermediate 60 . Aldol condensation proceeded stereoselectively to give exo-erythro isomer 64 as a major product (100\% exo-selective; erythro:threo at C1'-position=15:1). Addition of one carbon unit, selective ozonolysis and silylation gave lactol-ether 65. At this point we expected that hydroboration-oxidation should give the desired $\beta$-hydroxymethyl group, because the $\beta$-face is sterically congested by the lactol-ether ring. Unfortunately, the reaction gave a 1:1 mixture of two isomers, which was separated after oxidation of the lactol to give pure lactone 66. Hindered borane did not work at all. Hydroxylation of 66 with Davis' reagent afforded sorokinianin 60 exclusively. Protection of the primary $\mathrm{OH}$ group was essential for stereospecific hydroxylation. When it was executed without protection, only the epimeric hydroxyl group was introduced to give episorokinianin. A plausible mechanism may be that the hydroxide ion deprotonated at the carbon bearing the newly introduced hydroxyl group and then protonation occurred from the less hindered backside. Thus, we succeeded in the synthesis of sorokinianin and the absolute configuration was determined (ref. 32).

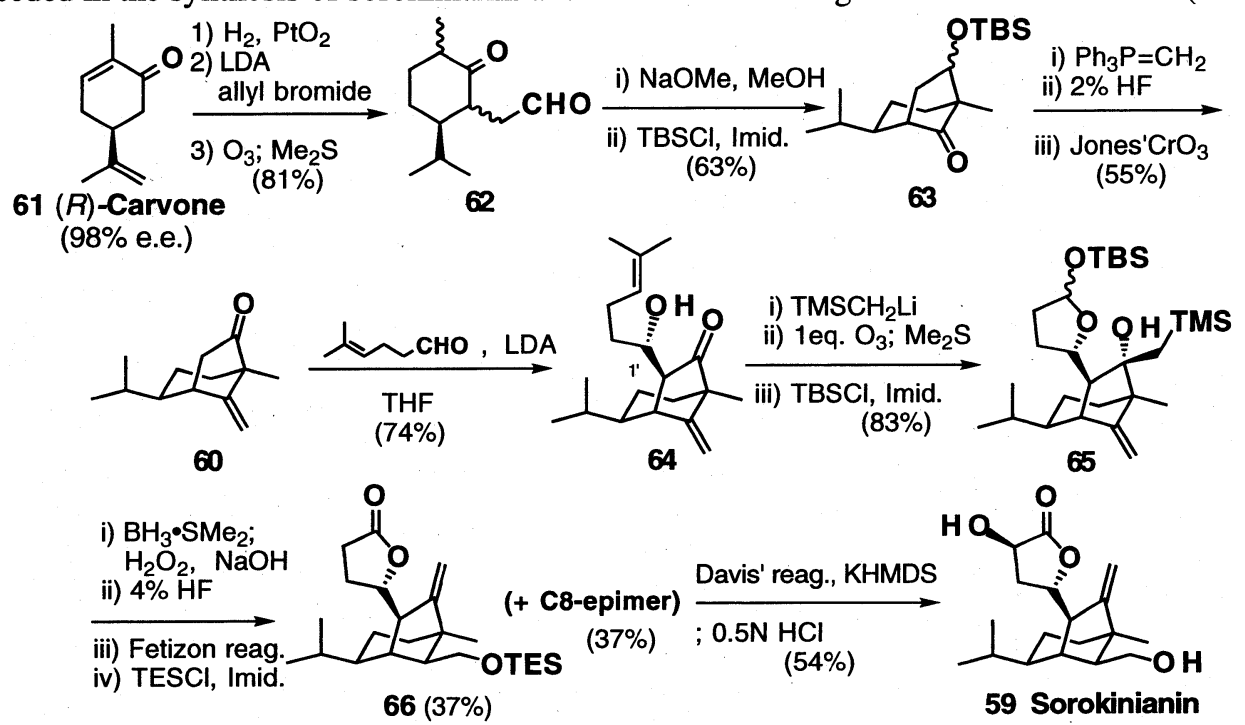

Fig. 15 Synthesis of Sorokinianin (Overall 17 steps, 3.4\%)

\section{Synthesis of Tanabalin: Insect Antifeedant}

Tanabalin 67 was isolated from a Brazilian medicinal plant, Tanacetum balsamita by Kubo, Kusumi and co-workers and the structure was elucidated unambiguously (ref. 33). This clerodane diterpenoid possesses strong antifeedant activity against a harmful pest, pink boll worm, Pectinophora

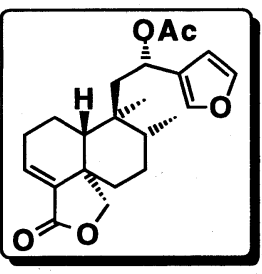

67 Tanabalin

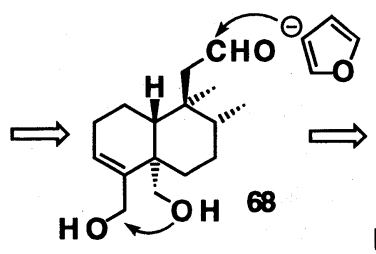

Lactone Formation<smiles>COO[C@@]12C(C(OC)OC)=CC(=O)C[C@]1(CC=C(C)C)[C@H](C)CC[C@@H]2C</smiles>

6

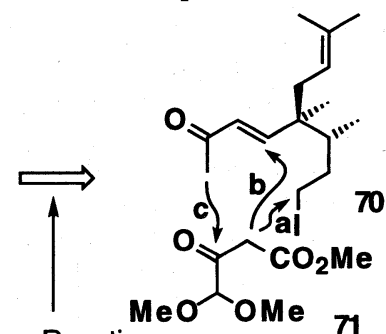

Tandem Reaction

Fig. 16 Synthesis of Tanabalin : Plan 
gossypiella. Our synthetic plan was to employ properly substituted trans-octalone 69 as a precursor, to which just addition of a furan ring and functional group modifications should give tanabalin. The key reaction was how to construct this trans-decalin skeleton and we adopted the tandem reaction to obtain the system in a single operation. Basic treatment of the known $\beta$-keto ester $\mathbf{7 1}$ with iodide $\mathbf{7 0}$ should effect initially alkylation (a) and successively intramolecular Robinson-type Michael and aldol reaction (b, c) to give the desired skeleton all at one time. In fact, this was the case under thermodynamic condition and we could complete the synthesis of tanabalin as follows.

We selected the known $\delta$-lactone $(R)-72$ as a starting material (ref. 34). Although the known procedures were biochemical transformations, we employed chemical transformation from $(S)$-citronellol via oxidative cleavage to $(R)-72$. Sequential alkylation of $(R)-72$ gave 73 stereospecifically. DIBAL reduction and the successive Horner-Wittig reaction gave the enone $\mathbf{7 4}$, which was converted to the corresponding iodide $\mathbf{7 0}$ in three steps. Bromide formation had to be done quickly after liberation of the hydroxyl group by fluoride treatment, or the formation of the substituted tetrahydropyran via cyclization was inevitable. The next tandem reaction was a critical step and the best result was obtained when a mixture of the iodide 70 and $\beta$-keto ester 71 was refluxed with sodium methoxide in methanol. The desired trans-octalone 69 was obtained as the sole product in $82 \%$ yield. In this tandem reaction, the second step, intramolecular Michael reaction of $75 \mathbf{a}$ to $\mathbf{7 5} \mathbf{b}$, determined the stereochemistry of the octalone 69. As designated like 75t, maximum overlap between HOMO of enolate and LUMO of enone might be generated when both chains are located at equatorial position, because the bulky prenyl group fixes the molecule by extending to the equatorial position at the transition state.

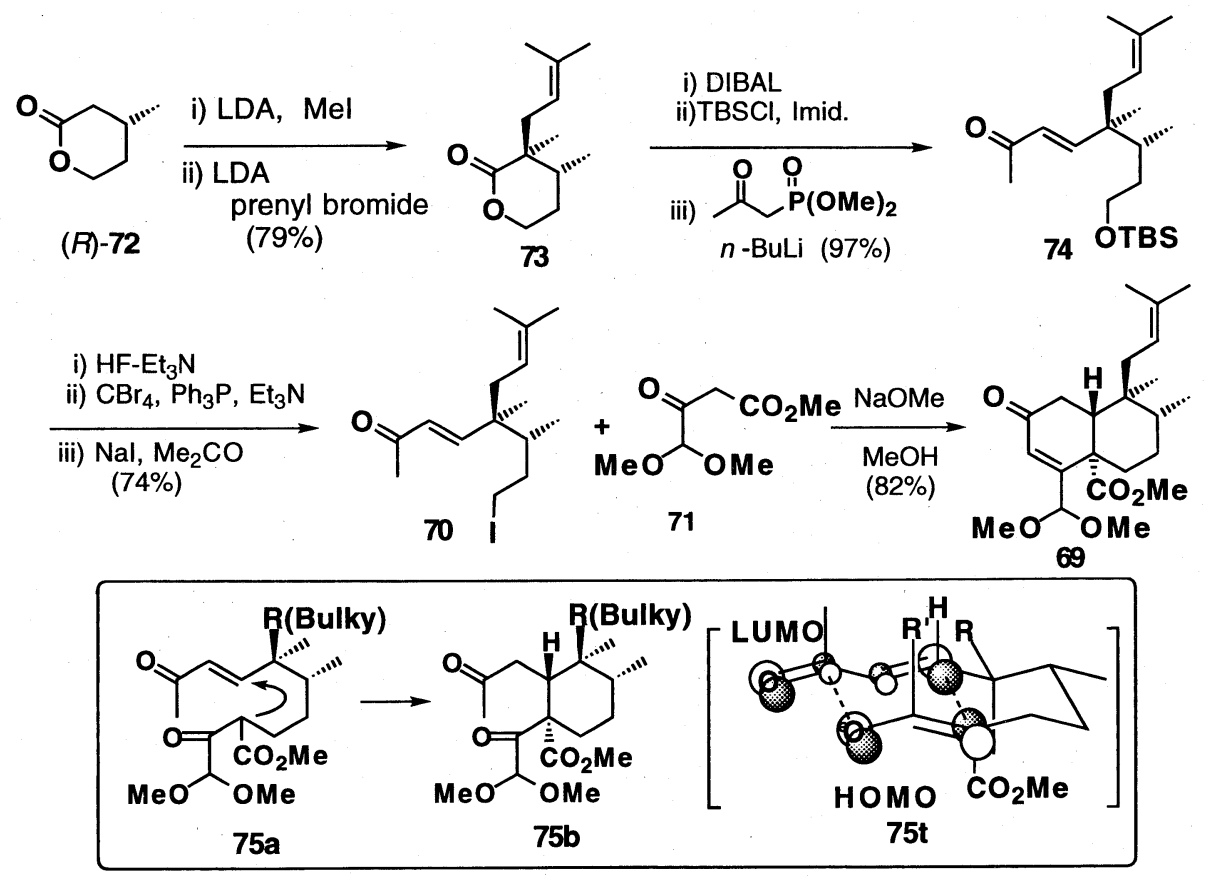

Fig. 17 Synthesis of (-)-Tanabalin : Tandem Cyclization

Now, the carbonyl group was reduced and removed by Barton's deoxygenation procedure via thiocarbamate 76. Acid hydrolysis of acetal and the following DIBAL reduction gave diol 7 7. Oxidation with Fetizon reagent directly gave the $\gamma$-lactone, which provided aldehyde $\mathbf{7 8}$ by careful ozonization. Reaction with 3-furylmagnesium bromide gave a mixture of epimeric alcohols 79 and 80 (1:1) in poor yield. The yield was much improved up to $68 \%$ using 3-furyllithium. Finally, acetylation of 79 afforded 
(-)-tanabalin, (-)-67. The epimer 80 must be convertible to tanabalin by Mitsunobu inversion with acetic acid. The synthesis of this clerodane diterpene was completed in only 18 steps (ref. 35 ) .

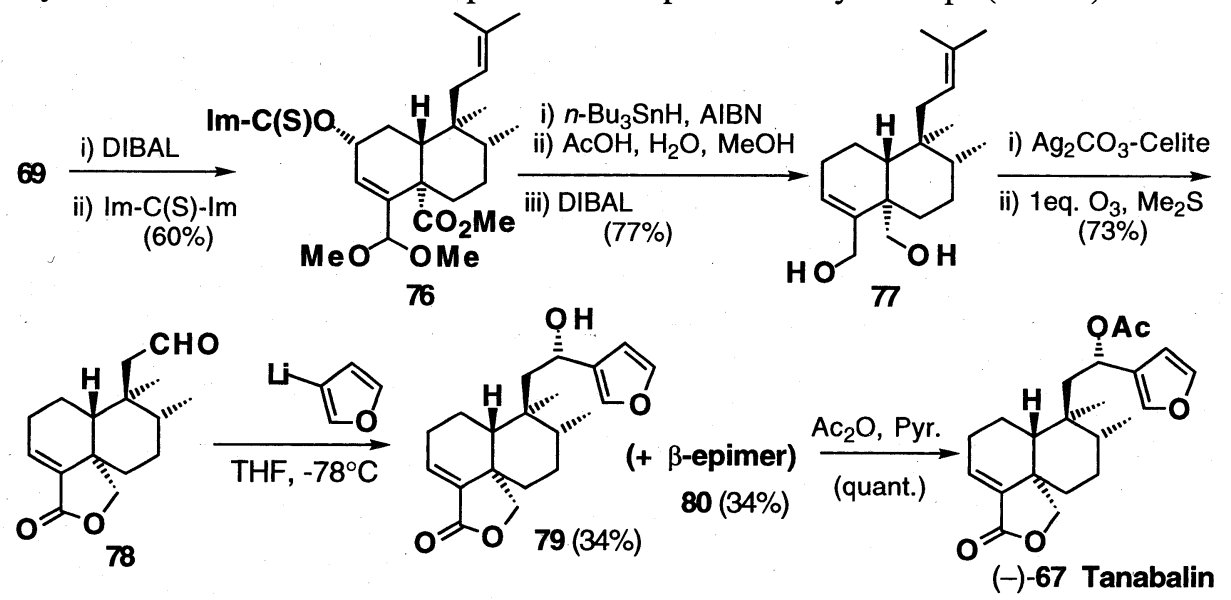

Fig. 18 Synthesis of (-)-Tanabalin (Overall 18 steps, 6\%)

\section{Conclusion}

We have been devoted to the synthesis of several bioregulators and related analogs with remarkable biological activities especially with regard to plant growth regulation, nutrition and physiology in optically active forms and have successfully developed general and efficient procedures. Application of these methods to afford substances should help further development of chemical and biological studies in plant physiology.

Acknowledgments: The authors would like to thank all of their co-workers whose names are cited in the references for their devotion to the synthetic work. We are much indebted to the Japanese Ministry of Education, Science, Culture and Sports, Japan Society for the Promotion of Science and T. Hasegawa Co., Ltd. for financial support. Generous gifts of starting materials from Fuji Pharmaceutical Industry Co., Ltd., Shiono Koryo Kaisha Ltd. and Takasago International Corporation are gratefully acknowledged.

\section{References and Notes}

(1) Demole, E.; Lederer, E.; Mercier, D. Helv. Chim. Acta 1962, 45, 675.

(2) Aldridge, D.C.; Galt, S.; Giles, D.; Turner, W.B. J. Chem. Soc., (C) 1971, 1623.

(3) Nishida, R.; Baker, T.C.; Roelofs, W.L. J. Chem. Ecol. 1982, 8, 947.

(4) Yoshihara, T.; Omer, E.-S.A.; Koshino, H.; Sakamura, S.; Kikuta, Y.; Koda, Y. Agric. Biol. Chem. 1989, 53, 2835.

(5) Matsuura, H.; Yoshihara, T.; Ichihara, A.; Kikuta, Y.; Koda, Y. Biosci. Biotech. Biochem. 1989, 53, 2835.

(6) Helmchen, G.; Goeke, A.; Lauer, G.; Urmann, M.; Fries, J. Angew. Chem. Int. Ed. Engl. 1990, 29, 1024.

(7) (a) Kitahara, T.; Nishi, T.; Mori, K. Tetrahedron 1991, 47, 6999. (b) Kitahara, T.; Warita, Y.; Abe, M.; Seya, M.; Takagi, Y.; Mori, K. Agric. Biol. Chem. 1991, 55, 1013.

(8) Kitahara, T.; Inoue, M.; Tamogami, S.; Kaiser, R. Tetrahedron 1996, 52, 1487. 
(9) Kaiser, R. The scent of orchids: Olfactory and chemical investigation, Elsevier, Amsterdam, 1993, 150-152, 210-212.

(10) Nishi, T.; Kitahara, T. Proc. Japan. Acad. 1995, 71B, 20.

(11) Inoue, M.; Kitahara, T. Tetrahedron submitted for publication.

(12) (a) Tanaka, S.; Wada, K.; Marumo, S.; Hattori, H. Tetrahedron Lett. 1984, 25, 5907. Tirilly, Y.; Kloosterman, J.; Sipma, G.; Kettenes-van den Bosch, J.J. Phytochem. 1983, 22, 2082. (b) Riche, C.; Pascard-Billy, C.; Devys, M.; Gaudemer, A.; Barbier, M.; Bousquet, J.F. Tetrahedron Lett. 1974, 2765. Bottalico, A.; Frisullo, S.; Lerario, P.; Randazzo, G.; Capasso, R. Phytopatho. Medit. 1982, 21, 39. (c) Kenfield, D.S.; Bunkers, G.; Wu, Y.-H.; Strobel, G.; Sugawara, F.; Hallock, Y.; Clardy, J. Experientia 1989, 45, 900. (d) Dhar, T.K.; Siddiqui, K.A.I.; Ali, E. Tetrahedron Lett. 1982, 23, 5459. (e) Sugawara, F.; Strobel, G.; Fisher, L.E.; Van Duyne, C.D.; Clardy, J. Proc. Natl. Acad. Sci. USA 1985, 82, 8291. (f) Guerriero, A.; D'Ambrosio, M.; Cuomo, V.; Vanzanella, F.; Pietra, F. Helv. Chim. Acta 1989, 72, 438.(g) Kim, S.-K.; Mizuno, K.; Hatori, M; Marumo, S. Tetrahedron Lett. 1994, 35, 1731.

(13) Lukes, R.M.; Poos, G.I.; Sarett, L.H. J. Am. Chem. Soc. 1952, 74, 1401.

(14) Kitahara, T.; Mori, K. Tetrahedron Lett. 1985, 26, 451.

(15) Kitahara, T.; Kurata, H.; Mori, K. Tetrahedron 1988, 44, 4339.

(16) Kitahara, T.; Kiyota, H.; Kurata, H.; Mori, K. Tetrahedron 1991, 47, 1649.

(17) Kitahara, T.; Touhara, K.; Watanabe, H.; Mori, K. Tetrahedron 1989, 45, 6387.

(18) Kitahara, T.; Akao, H., manuscript in preparation.

(19) Kobayashi, S.; Tsuchiya, K.; Harada, T.; Nishide, M.; Kurokawa, T.; Nakagawa, T.; Simada, N. J. Antibiot. 1994, 47, 697.

(20) Yoshida, T.; Koizumi, K.; Kawamura, Y.; Matsumoto, K.; Itazaki, H. Jpn. Pat. Kokaii 1993, 5310726.

(21) (a)Yasui, K.; Tamura, Y.; Nakatani, T.; Kawada, K.; Ohtani, M. J. Org. Chem. 1995, 60, 7567.

(b) Gurjar, M. K.; Henri Jr., J.T.; Bose, D.S.; Rao, A.V.R. Tetrahedron Lett. 1996, 37, 6615.; Gurjar, M. K.; Chakrabarti, A.; Rao, A.V.R. Heterocycles 1997, 45, 7. (c) Chida, N.; Yoshinaga, M.; Tobe, T. Ogawa, S. Chem. Commun. 1997, 1043.

(22) Watanabe, H.; Watanabe, H.; Kitahara, T. Tetrahedron Lett. 1998, 39. in press.

(23) Skrydstrap, T.; Bénéchie, M.; Khoung-Huu, F. Tetrahedron Lett. 1990, 31, 7145.

(24) Takai, K.; Nitta, K.; Utimoto, K. J. Am. Chem. Soc. 1986, 108, 7408.

(25) Mori, Y.; Suzuki, M. Tetrahedron Lett. 1989, 30, 4386.

(26) (a) Takagi, S. Soil Sci. Plant Nutr. 1976, 22, 423. (b) Takemoto, T.; Nomoto, K.; Fushiya, S.; Ouchi, R.; Kusano, G.; Hikino, H.; Takagai, S.; Matsuura, Y.; Kakudo, M. Proc. Japan. Acad. 1978, 54B, 249.

(27) Shioiri, T.; Hamada, Y.; Matsuura, F. Tetrahedron 1995, 51, 3939. and references cited therein.

(28) Klair, S.S.; Hindupur, M.R.; Kitahara, T. Tetrahedron Lett. 1998, 39, 89.

(29) Hindupur, M.R.; Osawa, H.; Kitahara, T. to be submitted.

(30) Nakajima, H.; Isomi, K.; Hamasaki, T.; Ichinoe, M. Tetrahedron Lett. 1994, 35, 9597.

(31) Corey, E.J.; Nozoe, S. J. Am. Chem. Soc. 1963, 85, 3527. ibid. 1965, 87, 5728.

(32) Watanabe, H.; Onoda, T.; Kitahara, T.; Mori, K. Tetrahedron Lett. 1997, 38, 6015.

(33) Kubo, I.; Jamalamadaka, V.; Kamikawa, T.; Takahashi, K.; Tabata, K.; Kusumi, T. Chem. Lett. 1996, 441.

(34) (a) Alvarez, E.; Cuvigny, T.; Hervé du Penhoat, C.; Julia, M. Tetrahedron 1988, 44, 111. (b) Poppe, L.; Novák, L.; Kolonitzs, P.; Bata, Á.; Szántay, C. Tetrahedron 1988, 44, 1447.

(35) Watanabe, H.; Onoda, T.; Kitahara, T. Tetrahedron Lett. submitted for publication. 